\title{
Wearable HD-DOT for investigating functional connectivity in the adult brain: a single subject, multi-session study
}

Uchitel, Julie, Blanco, Borja, Collins-Jones, Liam, VidalRosas, Ernesto, Cooper, Robert

Julie Uchitel, Borja Blanco, Liam Collins-Jones, Ernesto E. Vidal-Rosas, Robert J. Cooper, "Wearable HD-DOT for investigating functional connectivity in the adult brain: a single subject, multi-session study," Proc. SPIE 11920, Diffuse Optical Spectroscopy and Imaging VIII, 119201B (9 December 2021); doi: $10.1117 / 12.2615360$ 


\title{
Wearable HD-DOT for Investigating Functional Connectivity in the Adult Brain: A Single Subject, Multi- Session Study
}

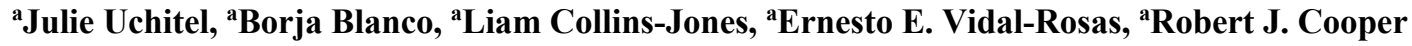 \\ ${ }^{a}$ DOT-HUB, Biomedical Optics Research Laboratory, Department of Medical Physics and Biomedical Engineering, University College \\ London, London, WC1E 6BT, UK
}

\begin{abstract}
We applied a wearable 24-module high-density diffuse optical tomography (HD-DOT) system in a resting state (RS) paradigm repeatedly in one subject. Seed-based correlation maps show large field-of-view RS functional connectivity.
\end{abstract}

\section{Background}

High-density diffuse optical tomography (HD-DOT) has been shown to approach the resolution and localization accuracy of BOLD-fMRI in the adult brain by exploiting densely spaced, overlapping samples of the probed tissue volume, but the technique has until recently required large and cumbersome optical fibre arrays[1]-[3]. The aim of the present study is to evaluate the use of a novel 24-module wearable HD-DOT system implementation that provides a comparable sampling density to large, fibre-based HD-DOT systems, but with vastly improved ergonomics.

\section{Materials and methods}

The HD-DOT device we applied is a 24-module LUMO system developed by Gowerlabs Ltd. The system consists of multiple, independent hexagonal modules (or 'tiles', Fig. 1a), each containing four photodiodes and three dualwavelength LEDs emitting at $735 \mathrm{~nm}$ and $850 \mathrm{~nm}$. The tiles are mounted into 'docks' (Fig. 1b), and a chain of docks is fitted into a neoprene cap. Fig. 1c shows a twenty-four-tile array fitted within a neoprene cap, designed to interrogate the occipital and frontal cortices. Fig. 1d shows the source and detector locations within these tiles projected onto the scalp surface mesh of the subject. The full arrangement yields a total of 3456 dual-wavelength source detector channels, which were sampled at a rate of $5 \mathrm{~Hz}$. Of this total number, approximately 1000 are expected to fall within the $10-45 \mathrm{~mm}$ range and thus provide viable signals.
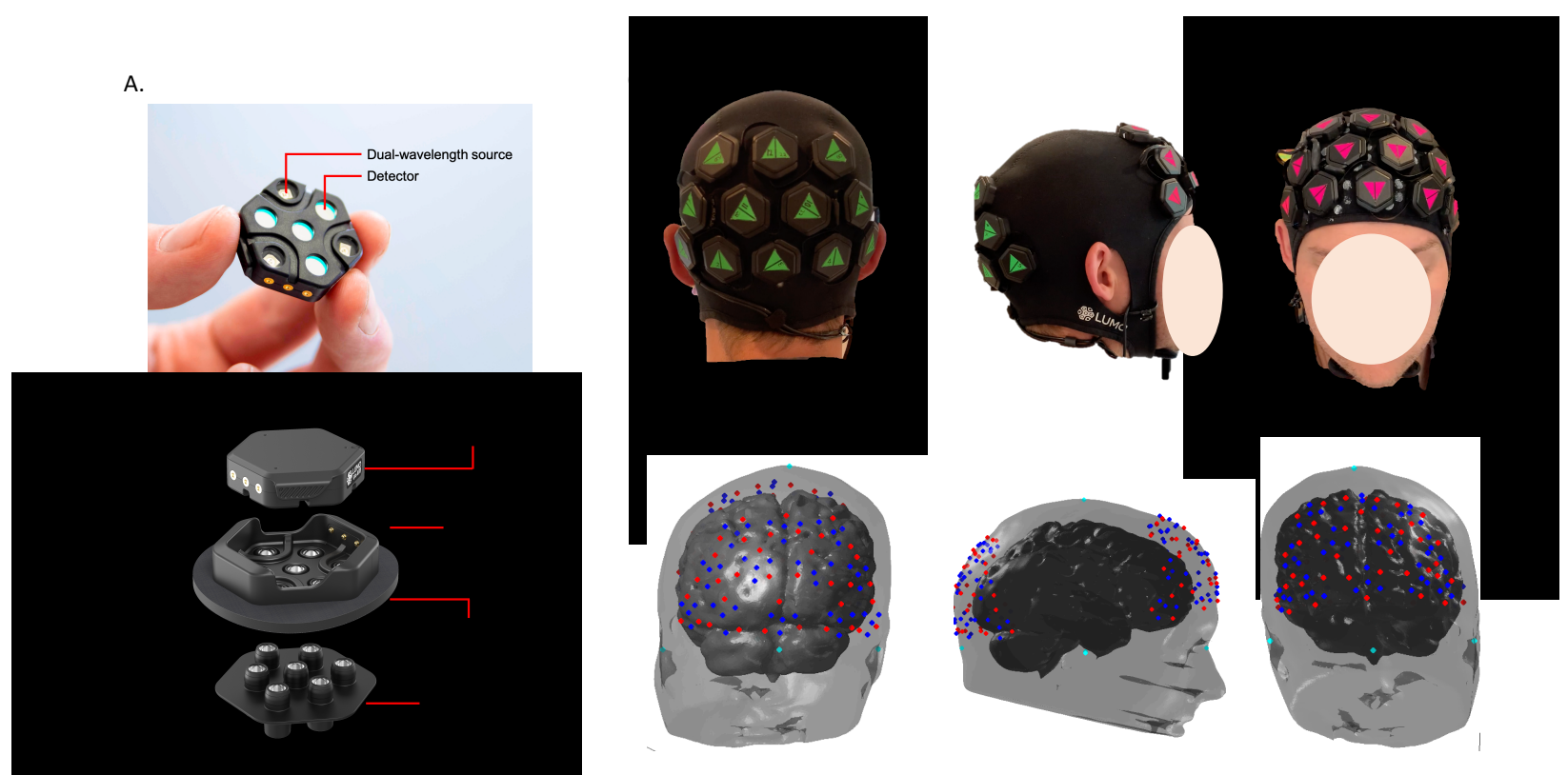

Fig. 1. (A) The LUMO tile module. (B) Assembly of complete LUMO module showing the tile, dock and light guides. (C) Neoprene cap fitted with a 24-tile LUMO system. (D) Image showing the location of the sources (red) and detectors (blue) on the scalp surface mesh of the subject. 


\subsection{Subject and experimental protocol}

The study considered a single, healthy participant (M, 36 years). Fifteen experimental sessions took place over a period of three weeks and under lockdown conditions due to the COVID-19 outbreak. Every recording was undertaken at the same time of day (between 11:00-13:00), in the same room with all conditions consistent. The cap was positioned on the subject to ensure that the same point on the cap was positioned over the inion, the distance of the cap above the ears was approximately equal on both sides, and the cap's front edge sat just above the eyebrows. The paradigm was 12 minutes of rest, in which the participant attended to a fixation cross displayed on a $50 \%$ grey background screen. Subject attention and alertness were monitored using a synchronised subject-facing webcam.

\subsection{Head Modeling and Optode Registration}

The 3D digitization of the optode locations was performed using photogrammetry. A short video of the subject wearing the cap was recorded in which the camera was panned around the stationary subject three times at different heights and downward angles. A 3D mesh model was created using the Metashape software package. A custommade program written in MATLAB allowed the manual selection of the locations of the cranial landmarks and tile markers (the corner of each of the pink and green triangles, see Fig. 1c) that were then used to determine the location of the sources and detectors on the subject's scalp. Structural MRI images of the participant were available from a previous experiment [4]. A head model was generated from these images which were used to create high-resolution tetrahedral mesh using Iso2mesh [http://iso2mesh.sourceforge net/cgi-bin/index.cgi].

\subsection{Signal Processing and Image Reconstruction}

In an initial assessment, channels were discarded based on minimum intensity values and their coefficient of variation. Intensity data were then converted to absorbance (O.D). Temporal filtering and global signal regression were performed simultaneously in a unique regression model. The average fNIRS signal across channels was included in the regression model, for $\mathrm{HbO}$ and $\mathrm{HbR}$ independently, to remove globally occurring hemodynamic signals[5].

The forward problem was modelled using the diffusion approximation[6]. The Jacobian matrix was calculated using Toast $++[7]$ via the finite element method (FEM). The optical properties of the tissue layers of the model at the wavelengths of interest were linearly interpolated from literature values for each tissue[8]. The Jacobian was calculated in a fine regular grid with size $30 \times 30 \times 30$ voxels and projected into the tetrahedral head model[9]. The changes in absorption coefficient were calculated using zeroth-order Tikhonov regularization. The recovered images of the change in absorption coefficient at the two wavelengths were then converted to images of change in oxy$(\mathrm{HbO})$ and deoxy-haemoglobin concentrations $(\mathrm{HbR})[10]$, and projected to the tetrahedral head model.

\subsection{Seed-based correlation}

To provide an initial determination of whether this experimental arrangement is capable of measuring functional connectivity, a seed-based correlation approach was used. In this approach, a manually selected node on the grey matter surface mesh of the subject was selected (as a 'seed' node), and the distance between this node and all other nodes in the mesh was calculated. If any node fell with within $5 \mathrm{~mm}$ of the seed node, it was considered as a part of the seed region. The $\mathrm{HbO}$ and $\mathrm{HbR}$ values in the reconstructed image for all the nodes in this seed region were then averaged to calculate an averaged seed-region signal. Correlation coefficients between the averaged seed region and all other nodes in the mesh were then calculated. This process was repeated for all sessions. The resulting correlation maps were then Z-transformed to allow for comparison across sessions. Z-transformed correlation maps from each session were then averaged to produce a preliminary group level correlation map.

\section{Results}

A $5 \mathrm{~mm}$ seed region was first selected for the lower right occipital cortex (Fig. 2a,b). Positive correlation contralateral to the seed region is apparent for the Z-transformed and averaged image (Fig 2a). The same results are displayed for HbR. (Fig 2b). A second $5 \mathrm{~mm}$ seed region was selected for the left prefrontal cortex (Fig. 2c,d). Similar to the findings from the seed in the occipital cortex, visual evaluation of the Z-transformed and averaged group correlation maps demonstrates positive correlation contralateral to the seed region for $\mathrm{HbO}$ and $\mathrm{HbR}$. 
A

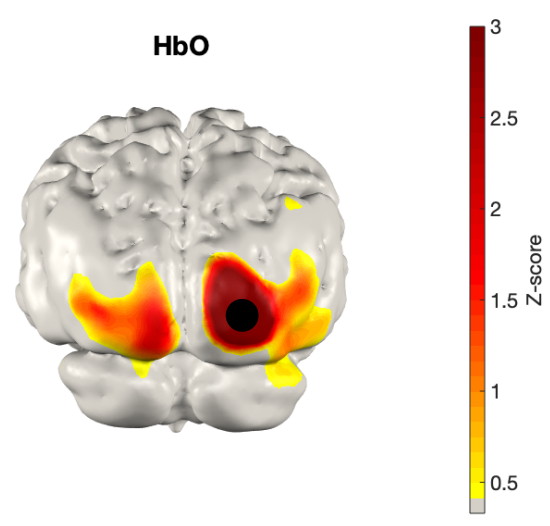

C.

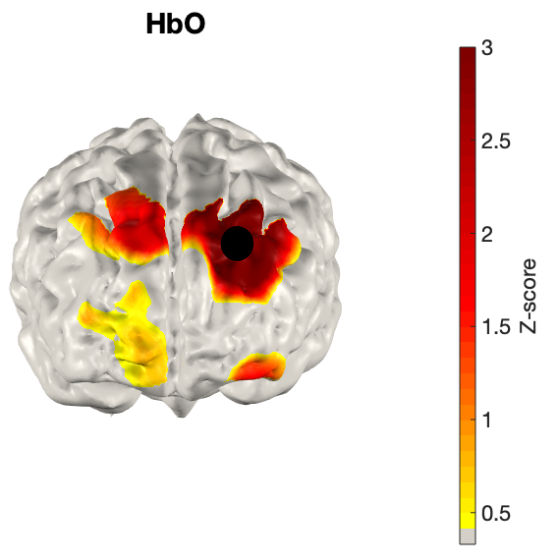

B.

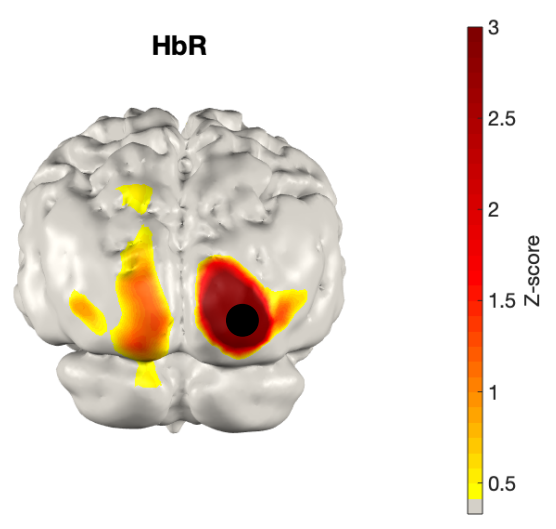

D.

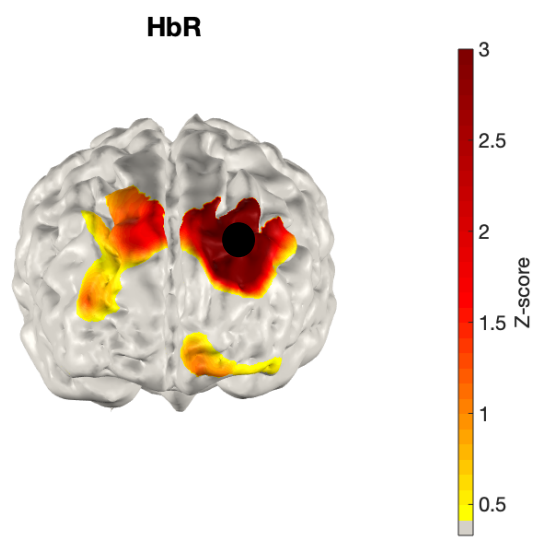

Fig. 2. Z-transformed, averaged group correlation maps with a seed in the lower right occipital cortex for $\mathrm{HbO}(\mathrm{A})$ and $\mathrm{HbR}$ (B) for all sessions. Another second seed was chosen for the upper left prefrontal cortex, and the Z-transformed, averaged group correlation maps is displayed for $\mathrm{HbO}(\mathrm{C})$ and $\mathrm{HbR}(\mathrm{D})$. All $\mathrm{Z}$-scores were thresholded to $\mathrm{Z}>0.3$. Seed regions are displayed as black circles.

\section{Future work}

In this work, we sought to demonstrate that our wide-field-of-view, 24-module configuration is able to capture basic features of resting state functional connectivity. Future analyses will include higher-level connectivity mapping over different cortical regions and explicit test-retest comparisons in this highly imaged individual dataset. Functional connectivity neuroimaging studies using a 12-tile LUMO system in the newborn population are also ongoing.

\section{References}

[1] B. W. Zeff, B. R. White, H. Dehghani, B. L. Schlaggar, and J. P. Culver, "Retinotopic mapping of adult human visual cortex with highdensity diffuse optical tomography," Proc Natl Acad Sci U S A, vol. 104, no. 29, pp. 12169-12174, Jul. 2007, doi: 10.1073/pnas.0611266104.

[2] B. R. White and J. P. Culver, "Quantitative evaluation of high-density diffuse optical tomography: in vivo resolution and mapping performance," J Biomed Opt, vol. 15, no. 2, p. 026006, Apr. 2010, doi: 10.1117/1.3368999.

[3] A. T. Eggebrecht et al., "A quantitative spatial comparison of high-density diffuse optical tomography and fMRI cortical mapping," Neuroimage, vol. 61, no. 4, pp. 1120-1128, Jul. 2012, doi: 10.1016/j.neuroimage.2012.01.124.

[4] H. Zhao et al., "A wide field-of-view, modular, high-density diffuse optical tomography system for minimally constrained three-dimensional functional neuroimaging," Biomed. Opt. Express, BOE, vol. 11, no. 8, pp. 4110-4129, Aug. 2020, doi: 10.1364/BOE.394914.

[5] I. Tachtsidis and F. Scholkmann, "False positives and false negatives in functional near-infrared spectroscopy: issues, challenges, and the way forward," Neurophotonics, vol. 3, no. 3, p. 031405, Jul. 2016, doi: 10.1117/1.NPh.3.3.031405.

[6] S. R. Arridge, “Optical tomography in medical imaging," Inverse Problems, vol. 15, no. 2, pp. R41-R93, Apr. 1999, doi: 10.1088/0266$5611 / 15 / 2 / 022$.

[7] M. Schweiger and S. Arridge, "The Toast++ software suite for forward and inverse modeling in optical tomography," J Biomed Opt, vol. 19, no. 4, p. 040801, Apr. 2014, doi: 10.1117/1.JBO.19.4.040801.

[8] F. Bevilacqua, D. Piguet, P. Marquet, J. D. Gross, B. J. Tromberg, and C. Depeursinge, "In vivo local determination of tissue optical properties: applications to human brain," Appl Opt, vol. 38, no. 22, pp. 4939-4950, Aug. 1999, doi: 10.1364/ao.38.004939.

[9] M. Schweiger and S. Arridge, "Image reconstruction in optical tomography using local basis functions," J. Electronic Imaging, vol. 12, pp. 583-593, Oct. 2003, doi: 10.1117/1.1586919.

[10]M. Cope, "The development of a near infrared spectroscopy system and its application for non invasive monitoring of cerebral blood and tissue oxygenation in the newborn infants," Doctoral, University of London, 1991. 